\title{
Evaluation of Inter-Occasion Variability on Trospium Pharmacokinetics in Healthy Human Subjects using Non-Compartment Methods
}

\author{
Sundara Moorthi Nainar Murugesan*, Ravisekhar Kasibhatta, Prabakaran Desomayandhan, Saji Vijayan, Vijay Tate, Hemlata Nigam, \\ Ashish Saxena, Praveen Kumar Vittala and Sikandar Ali Khan
}

Lupin Ltd, Pune, Maharashtra India

\begin{abstract}
Objective: The main objective of this study was to assess the impact of inter-occasion variability (IOV) on Trospium plasma concentration level from typical crossover pharmacokinetic study using non-compartment model analysis.

Methods: An open, randomized, fasting, single-dose, two way crossover reference replicate study was performed with 36 healthy, non-smoking, male subjects. Plasma concentration of Trospium was estimated. The influence of inter-occasion on Trospium pharmacokinetics was evaluated using non-compartment model analysis.

Results: Results from the non-compartment analyses showed that inter-occasion variability as measured by coefficient of variation was found more than $30 \%$ for pharmacokinetic parameters $C_{\max }$, AUClast and $\mathrm{Vd} / \mathrm{F}$. In addition, IOV for $\mathrm{C}_{\max }$ was higher when compared with any other pharmacokinetic parameters.

Conclusion: Overall, considerable variability associated with Trospium chloride pharmacokinetics between occasions was established. Trospium was well tolerated in the treated subjects and there was no serious adverse event noted in the entire study. Thus, major differences in obtained variability seen in this study are likely to be of only limited clinical significance.
\end{abstract}

Keywords: Cholinergics; Crossover design; Healthy volunteers; Trospium chloride

\section{Introduction}

Trospium Chloride is an established anti-cholinergic compound used for the long-term treatment of overactive bladder. Trospium plasma levels are characterized by a great inter-individual and intraindividual variability $[1,2]$. The reported Trospium intra-subject variability is $72 \%$ and of $60 \%$, for AUC and $\mathrm{C}_{\max }$, respectively [3]. Trospium chloride exhibits diurnal variability in exposure with a decrease of both $\mathrm{C}_{\max }$ and AUC for evening dosing relative to morning dose [4-6]. Of interest, there appears to be circadian variability in trospium chloride pharmacokinetics, with a decrease in $\mathrm{C}_{\max }$ of up to $59 \%$ and AUC of up to $33 \%$ for evening dosing relative to morning dosing [7]. Also, the inter-individual variability in pharmacokinetics was more pronounced during the morning dose administration interval compared with the evening dose administration interval. Reported mean coefficient of variation of $42 \%$ and $33 \%$ for AUC-ss and $46 \%$ and $35 \%$ for $\mathrm{C}_{\max }$-ssat steady state is reported for the morning dose and the evening dose, respectively [8].

Variability is an inherent property and it can be decreased or theoretically eliminated by implementing "ideal" experiments and dataprocessing techniques [9]. All drugs exhibit between-subject variability in exposure and response and many studies performed during drug development are aimed at identifying and quantifying this variability to improve the safety and efficacy of a drug agent. Variability is usually characterized in terms of fixed and random effects. The fixed effects are the population average values of pharmacokinetic parameters. The random effects quantify the amount of pharmacokinetic variability that is not explained by the fixed effects. These random effects subsets are inter-subject variability, inter-occasion variability and intra-individual and residual variability. Lack of separation of inter-occasion variability from residual intra-subject variability may produce bias in estimation of population pharmacokinetic parameters [10]. Describing betweenoccasion variability can be an important tool for identifying underlying reasons for altered response over time [11]. Since, individual pharmacokinetic parameters may change randomly between study occasions and analysis of simulated data shows that ignoring such inter-occasion variability (IOV) may result in biased population parameter estimates. Neglecting IOV also results in a high incidence of statistically significant spurious period effects [12]. The identification and quantification of covariates, particularly using population pharmacokinetics is now seen as an integral part of drug development. Many pharmaceutical companies go through unnecessary cycles of clinical studies involving formulation optimization without attention to the feasibility of reducing inter-individual variability and the source of such variation [13].

Recent years, different types of pharmacokinetic modeling approaches were evaluated to quantify the variability of Trospium pharmacokinetics, however to the best of our knowledge pharmacokinetic approaches related to treatment occasion were still not yet evaluated for Trospium. Thus, present study was aimed to assess the impact of inter-occasion variability on Trospium plasma concentration level from typical crossover pharmacokinetic study using a method which did not involve any assumption related to the evaluation of pharmacokinetics, i.e., non-compartment model analysis.

*Corresponding author: Sundara Moorthi Nainar Murugesan Lupin Ltd, Pune, Maharashtra, India, Tel: +91-9766483978, E-mail: sundaramuurugesan@lupinpharma.com

Received August 26, 2014; Accepted October 04, 2014; Published October 31 2014

Citation: Murugesan SMN, Kasibhatta R, Desomayandhan P, Vijayan S, Tate V, et al. (2014) Evaluation of Inter-Occasion Variability on Trospium Pharmacokinetics in Healthy Human Subjects using Non-Compartment Methods. J Bioequiv Availab 6 202-205. doi:10.4172/jbb.1000205

Copyright: () 2014 Murugesan SMN, et al. This is an open-access article distributed under the terms of the Creative Commons Attribution License, which permits unrestricted use, distribution, and reproduction in any medium, provided the original author and source are credited. 


\section{Materials and Methods}

All materials used in this study were complied with current United States Pharmacopeia (USP) compendial specifications.

\section{Formulation}

SANCTURA XR ${ }^{\mathrm{TM}}$ (Trospium Chloride Extended Release Capsules) $60 \mathrm{mg}$ manufactured by Catalent Pharma solutions, Inc., Somerset, NJ 08873 USA and manufactured for Allergan, Inc., Irvine, CA 92612 USA.

\section{Treatment occasion}

Subjects were exposed with single oral dose of Sanctura $\mathrm{XR}^{\mathrm{TM}}$ (Trospium Chloride extended release capsules) $60 \mathrm{mg}$ in two treatment occasions and both occasions were separated by interval of 14 days from the completion of dosing in one occasion to the start of the dosing in the next occasion.

\section{Pharmacokinetic study}

Selection of study population: All subjects underwent a screening procedure. Medical history and detailed demographic data were recorded. Each subject underwent a complete general physical examination and laboratory tests of hematology, biochemistry, urine analysis and serology. Only medically healthy subjects with clinically acceptable laboratory profiles, ECG and chest X-ray were enrolled into the study. Further, the subjects were selected on the basis of inclusion and exclusion criteria. Crossover design has been selected in order to minimize the variability due to subject specific parameters.

Study design: An open, randomized, fasting, single-dose, two way crossover reference replicate study was performed with 36 healthy, non-smoking, male subjects. The study protocols for Trospium were approved by the Institutional Ethics Committee at the clinical site (approval no. TROS/395/09). The study was conducted in accordance with the ethical principles that have their origin in the Declaration of Helsinki and in compliance with ICH-GCP. Written informed consent was obtained from all subjects prior to enrolment in the study.

In each study period, after an overnight fast of at least $10 \mathrm{hrs,}$ as per the randomization schedule single oral dose of Trospium Chloride extended release capsules $60 \mathrm{mg}$ (Sanctura $\mathrm{XR}^{\mathrm{TM}}$ ) was orally administered with $240 \mathrm{~mL}$ of drinking water in sitting posture at ambient temperature in the morning. A total of 23 blood samples were collected in each period. The pre-dose blood sample $(1 \times 5 \mathrm{~mL})$ was collected within 1 hour prior to dosing. The post-dose blood samples $(1 \times 5-\mathrm{mL}$ each) were collected at 1.00, 2.00, 2.50, 3.00, 3.50, 4.00, 4.50, 5.00, 5.50, $6.00,6.50,7.00,7.50,8.00,9.00,10.00,12.00,16.00,24.00,48.00,72.00$ and 96.00 hours after dosing. Subjects were seated upright position for the first two hours following drug administration and prohibited from any strenuous or athletic activity during housing period of the study. Plasma samples were stored at $-50^{\circ} \mathrm{C}$ before analysis. Plasma samples were separated and analyzed for Trospium plasma concentrations.

\section{Analytical methods}

A highly sensitive, specific and evaporation free SPE extraction, LC-MS/MS method has been developed for the estimation of Trospium in human $\mathrm{K}_{3}$ EDTA plasma. The analyte was separated using isocratic mobile phase $\{$ Methanol: Ammonium formate $(60: 40 \mathrm{v} / \mathrm{v})\}$ on reverse phase column and analyzed by MS/MS in the multiple reaction monitoring mode using the respective $[\mathrm{M}(+)]$ cations, $\mathrm{m} / \mathrm{z}$ 392.3-182.1 for Trospium. The total chromatographic run time was $3.50 \mathrm{~min}$. The developed method was validated in human plasma with a lower limit of quantification of $0.0560 \mathrm{ng} / \mathrm{mL}$. A linear response function was established for the range of concentrations $0.0560-13.2960 \mathrm{ng} / \mathrm{mL}$ for Trospium in human plasma. The intra- and inter-day precision values for Trospium met the acceptance as per USFDA guidelines. Trospium was stable in the series of stability studies viz., bench-top, auto-sampler, dry extracts and freeze/thaw cycles.

\section{Data analysis method}

The influence of inter-occasion, inter-individual and residual variability on Trospium pharmacokinetics was evaluated using noncompartment model analysis as detailed below. Non-compartment model analysis (NCA) was used to reveal the pharmacokinetics of Trospium, because NCA model did not involve any assumption related to the evaluation of pharmacokinetics. Trospium plasma concentration versus time data were evaluated using the Phoenix Win Nonlin software version 6.3. The pharmacokinetic parameters $C_{\text {max }}$, AUClast, AUCinf, Kel, Vd/F and CL/F for Trospium were calculated by non-compartmental method using Phoenix WinNonlin Software (version 6.3). All calculations of non-compartmental analysis were based on actual sampling times. The pharmacokinetic parameters $\mathrm{C}_{\max }$, the maximum observed concentration, AUC, the area under the concentration time curve, Kel, elimination rate constant, $\mathrm{Vz} / \mathrm{F}$, Volume of distribution and $\mathrm{Cl} / \mathrm{F}$, Clearance were determined for each occasion. For extravascular models the fraction of dose absorbed cannot be estimated, therefore Volume and Clearance for these models are actually $\mathrm{Vz} / \mathrm{F}$ or $\mathrm{Cl} / \mathrm{F}$ where $\mathrm{F}$ is the fraction of dose absorbed. To calculate the elimination rate constant $(\mathrm{Kel})$, regression analyses were performed on the natural $\log (\mathrm{Ln})$ of plasma concentration values (Y-axis) versus time (X-axis). Calculations were made between a time point where log-linear elimination phase begins and the time at which the last concentration above the limit of quantitation occurred. Area under the concentration - time curves (AUC) was calculated using the linear trapezoidal rule. Volume of distribution is estimated based on the terminal phase. Total clearance was calculated using the formula $\mathrm{CL}=$ Dose/AUC. Also, intra-individual, inter-individual and residual variability were calculated. Obtained results for intra-individual was considered as inter-occasion variability since, subjects and formulations remain same in both period-I and period-II. Linear mixed effect models used to model the $\log$ transformed $\mathrm{C}_{\max }$, AUClast, AUCinf, Kel, Vd/F and $\mathrm{CL} / \mathrm{F}$ for Trospium as functions of sequence, treatment and period, as fixed effects, and subjects nested within sequence as a random effect. The default model $\operatorname{Var}(\operatorname{Subj}(\operatorname{Seq})$ ) was the inter-individual (between subjects) variance, and residual variance is the intra-individual (within subject) variance

\section{Results}

A total of 36 healthy adult human male subjects were enrolled the study and 25 subjects completed the study. One subject withdrawn his consent for further participation, one subject withdrawn due to adverse event and nine subjects were not reported after period-I dosing. Plasma samples from 25 subjects were analyzed to determine the plasma concentrations of Trospium. Non-compartmental analyses were performed on data obtained from 25 subjects, who completed the study as per the protocol. Baseline characteristics of subjects who completed the study and included for pharmacokinetic analysis are presented in Table 1 .

The individual concentration time course of Trospium was derived from observed plasma concentrations. No significant time dependent changes in pharmacokinetics of Trospium between two different occasions (Figure 1) were visible in the graphical assessment of time 


\begin{tabular}{|c|c|}
\hline \multirow{2}{*}{ Parameters } & Mean \pm SD (Range) \\
\cline { 2 - 2 } & $\mathbf{( N = 2 5 )}$ \\
\hline Age (years) & $26.64 \pm 6.62(18-43)$ \\
\hline BMI (kg/m $)$ & $21.86 \pm 2.11(19.25-24.62)$ \\
\hline Height $(\mathbf{c m})$ & $164.40 \pm 7.42(150-178)$ \\
\hline Weight $(\mathbf{k g})$ & $59.16 \pm 7.75(50-77)$ \\
\hline
\end{tabular}

Table 1: Subjects' baseline characteristics.

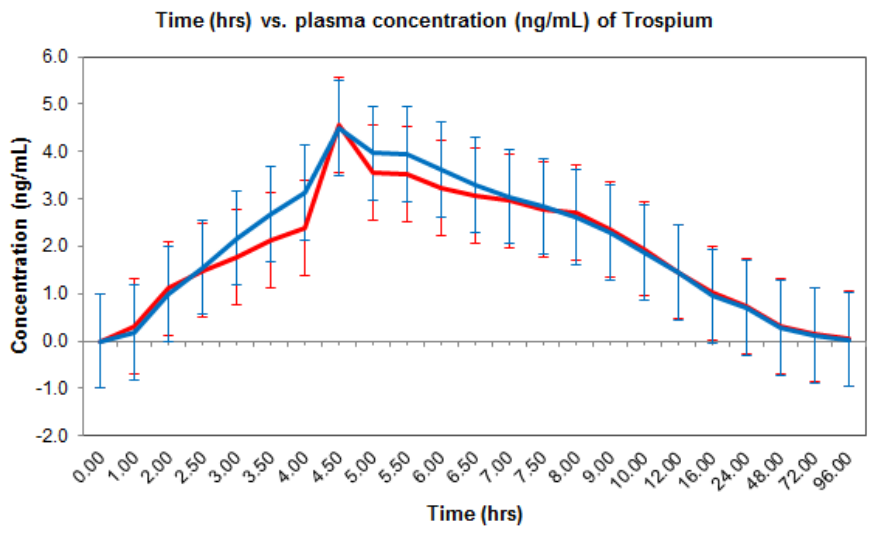

- Occasion-1 Occasion-2

Figure 1:

versus plasma concentration profile. In present study it was evidencing that maximum absorption for Trospium was observed from $4.5 \mathrm{hrs}$ - $6.50 \mathrm{hrs}$. After $6.50 \mathrm{hrs}$ there was a constant decline in the plasma concentration in absorption of Trospium was observed.

The Mean and Coefficient of Variation $(\mathrm{CV})$ were calculated for plasma concentrations of Trospium for each sampling time and occasion. Results are presented in Table 2.

Recognition of IOV essentially dissects the first type of variation (parameter variation) into two parts: between individual and between occasion. It is important to remember that total variation is preserved, so that if IOV is unrecognized, it inflates either IIV or residual variability [12]. Results of mean pharmacokinetic parameters are presented in Table 3.

Results from the NCA analyses showed that inter-occasion variability as measured by coefficient of variation was found more than $30 \%$ for pharmacokinetic parameters $\mathrm{C}_{\max }$, AUC last and $\mathrm{Vd} / \mathrm{F}$. However, when consider the amount of obtained residual variability, except $\mathrm{C}_{\max }$ overall IOV may be less for other reported PK parameters. Since, the IOV of $\mathrm{C}_{\max }$ was found to be $26.27 \%$ even after excluding the residual variability from IOV.

Further, estimation of inter-individual variability data demonstrated that consistent variability between occasion 1 and 2 (i.e., $77.22 \%$ vs. $73.22 \%$ ) was observed for $\mathrm{C}_{\max }$. While, inter-individual variability for pharmacokinetic parameters, AUC (i.e., $53.83 \%$ vs $60.40 \%$ ), AUCinf (i.e., $45.47 \%$ vs. $52.42 \%$ ), $K e l$ (i.e., $27.56 \%$ vs. $41.03 \%$ ), $\mathrm{Vd} / \mathrm{F}$ (i.e., $53.70 \%$ vs. $76.69 \%$ ) and $\mathrm{CL} / \mathrm{F}($ i.e., $59.85 \%$ vs. $71.06 \%$ ) was not consistent between two treatment occasions. These results evidencing that those pharmacokinetic parameters estimation involved over the time period was observed with incoherent inter-individual variability and $\mathrm{C}_{\max }$ showed consistent inter-individual variability may due to single point estimation.
In addition, IOV for $\mathrm{C}_{\max }$ was higher when compared with any other pharmacokinetic parameters, hence we suspect the residual error associated with $\mathrm{C}_{\max }(17.60 \%)$ may result increase in IOV.

\section{Discussion}

In general trospium chloride is reported as slow absorption and low absolute bioavailable drug. Absorption of trospium chloride has been measured in a number of separate pharmacokinetic studies undertaken with healthy male volunteers [14-16].

The present approach reduces the complex inter-occasion variability modeling processes using non-linear mixed effect model. Using non-compartment model the possible inter-occasion variability which results from the residual variability was explained. Replicating the dose administration will have an advantage to rule out the subject specific parameter influence on obtained pharmacokinetic parameters.

Doroshyenko et al., described that regarding the level of absorption of Trospium an influence of the reduced $\mathrm{pH}$ in the stomach would not be expected, given that trospium chloride is a highly soluble drug according to the BCS concept and almost exclusively (though incompletely) absorbed in the small intestine. They suspected about the existence of an enterohepatic circulation of trospium chloride [8]. In present study the maximum absorption for Trospium was observed from $4.50 \mathrm{hrs}-6.50 \mathrm{hrs}$, and after that there was a constant decline in the plasma concentration curve demonstrated that $\mathrm{pH}$ related influence was not expected during the absorption phase of Trospium.

\begin{tabular}{|c|c|c|c|c|c|c|}
\hline $\begin{array}{l}\text { Time } \\
\text { (hrs) }\end{array}$ & $\begin{array}{l}\text { Clock } \\
\text { time }\end{array}$ & $\begin{array}{c}\text { Collection } \\
\text { day }\end{array}$ & Occasion-1 & $\% \mathrm{CV}$ & Occasion-2 & $\% \mathrm{CV}$ \\
\hline 0.00 & 8:00 AM & 1 & 0.0000 & 0.0000 & 0.0000 & 0.0000 \\
\hline 1.00 & 9:00 AM & 1 & 0.3091 & 204.80 & 0.1788 & 142.00 \\
\hline 2.00 & 10:00 AM & 1 & 1.1097 & 123.20 & 0.9926 & 84.00 \\
\hline 2.50 & 10:50 AM & 1 & 1.4920 & 111.70 & 1.5576 & 70.10 \\
\hline 3.00 & 11:00 AM & 1 & 1.7780 & 93.80 & 2.1709 & 73.30 \\
\hline 3.50 & $11: 30 \mathrm{AM}$ & 1 & 2.1203 & 81.70 & 2.6864 & 76.30 \\
\hline 4.00 & 12 Noon & 1 & 2.3952 & 74.50 & 3.1227 & 82.20 \\
\hline 4.50 & 12.30 PM & 1 & 4.5560 & 87.10 & 4.4845 & 73.30 \\
\hline 5.00 & 1:00 PM & 1 & 3.5573 & 69.00 & 3.9609 & 71.30 \\
\hline 5.50 & 1:30 PM & 1 & 3.5252 & 66.10 & 3.9403 & 70.60 \\
\hline 6.00 & 2:00 PM & 1 & 3.2250 & 66.20 & 3.6200 & 67.80 \\
\hline 6.50 & 2:30 PM & 1 & 3.0602 & 64.30 & 3.3032 & 64.80 \\
\hline 7.00 & 3:00 PM & 1 & 2.9542 & 67.10 & 3.0475 & 64.00 \\
\hline 7.50 & 3:30 PM & 1 & 2.7650 & 69.30 & 2.8326 & 62.30 \\
\hline 8.00 & 4:00 PM & 1 & 2.7129 & 72.20 & 2.6067 & 64.20 \\
\hline 9.00 & 5:00 PM & 1 & 2.3614 & 87.60 & 2.2809 & 65.70 \\
\hline 10.00 & 6:00 PM & 1 & 1.9462 & 87.10 & 1.8579 & 69.30 \\
\hline 12.00 & 8:00 PM & 1 & 1.4573 & 68.60 & 1.4346 & 59.20 \\
\hline 16.00 & 12:00 AM & 1 & 1.0114 & 54.10 & 0.9452 & 53.50 \\
\hline 24.00 & 8:00 AM & 2 & 0.7350 & 38.30 & 0.6929 & 47.30 \\
\hline 48.00 & 8:00 AM & 3 & 0.3033 & 46.10 & 0.2673 & 59.90 \\
\hline 72.00 & 8:00 AM & 4 & 0.1328 & 46.80 & 0.1149 & 63.30 \\
\hline 96.00 & 8:00 AM & 5 & 0.0465 & 116.30 & 0.0339 & 138.30 \\
\hline \multicolumn{3}{|c|}{$\mathbf{N}$} & 23 & 23 & 23 & 23 \\
\hline \multicolumn{3}{|c|}{ Mean } & 1.8937 & 78.0826 & 2.0058 & 70.5522 \\
\hline \multicolumn{3}{|c|}{ SD } & 1.2963 & 38.3438 & 1.4176 & 27.2873 \\
\hline \multicolumn{3}{|c|}{ Min } & 0.0000 & 0.0000 & 0.0000 & 0.0000 \\
\hline \multicolumn{3}{|c|}{ Median } & 1.9462 & 69.3000 & 2.1709 & 67.8000 \\
\hline \multicolumn{3}{|c|}{ Max } & 4.5560 & 204.8000 & 4.4845 & 142.0000 \\
\hline \multicolumn{3}{|c|}{ Geometric Mean } & 1.3309 & 76.1399 & 1.3120 & 71.2128 \\
\hline
\end{tabular}

Table 2: Time-wise mean plasma concentration data of Trospium for Ocassion-1 and 2 . 
Citation: Murugesan SMN, Kasibhatta R, Desomayandhan P, Vijayan S, Tate V, et al. (2014) Evaluation of Inter-Occasion Variability on Trospium Pharmacokinetics in Healthy Human Subjects using Non-Compartment Methods. J Bioequiv Availab 6: 202-205. doi:10.4172/jbb.1000205

\begin{tabular}{|c|c|c|c|c|c|c|}
\hline \multirow[b]{2}{*}{ PK Parameters } & \multicolumn{5}{|c|}{ Mean \pm SD (Range) N=25 } & \multirow[b]{2}{*}{ Residual variability } \\
\hline & $\begin{array}{l}\text { Occasion-1 } \\
(N=25)\end{array}$ & $\begin{array}{l}\text { IIV } \\
(\% \mathrm{CV})\end{array}$ & $\begin{array}{l}\text { Occasion-2 } \\
(\mathrm{N}=25)\end{array}$ & $\begin{array}{c}\text { IIV } \\
\text { (\%CV) }\end{array}$ & $\begin{array}{c}\text { IOV } \\
(\% \mathrm{CV})\end{array}$ & \\
\hline $\begin{array}{c}C_{\max } \\
(\mathbf{n g} / \mathrm{mL})\end{array}$ & $\begin{array}{l}5.3 \pm 4.1 \\
(0.6-16.8)\end{array}$ & 77.22 & $\begin{array}{c}4.8 \pm 3.5 \\
(0.6-15.7)\end{array}$ & 73.22 & 43.87 & 17.60 \\
\hline $\begin{array}{c}\text { AUClast } \\
\text { (ng*hr/mL) }\end{array}$ & $\begin{array}{l}55.8 \pm 30.0 \\
(7.1-128.0)\end{array}$ & 53.83 & $\begin{array}{l}50.6 \pm 30.6 \\
(10.6-22.6)\end{array}$ & 60.40 & 33.07 & 10.38 \\
\hline $\begin{array}{c}\text { †AUCinf } \\
\text { (ng*hr/mL) }\end{array}$ & $\begin{array}{c}61.4 \pm 27.9 \\
(17.8-129.1)\end{array}$ & 45.47 & $\begin{array}{c}55.8 \pm 29.2 \\
(13.5-125.0)\end{array}$ & 52.42 & 24.11 & 5.65 \\
\hline $\begin{array}{l}\text { †Kel } \\
(1 / \mathrm{hr})\end{array}$ & $\begin{array}{c}0.040 \pm 0.01 \\
(0.03-0.06)\end{array}$ & 27.56 & $\begin{array}{l}0.04 \pm 0.01 \\
(0.02-0.08)\end{array}$ & 41.03 & 23.24 & 5.26 \\
\hline $\begin{array}{c}+V d / F \\
\text { (L) }\end{array}$ & $\begin{array}{l}33.9 \pm 18.20 \\
(7.19-77.22)\end{array}$ & 53.70 & $\begin{array}{c}38.3 \pm 29.34 \\
(9.35-139.37)\end{array}$ & 76.69 & 30.40 & 8.84 \\
\hline $\begin{array}{c}\text { †CL/F } \\
(\mathrm{mL} / \mathrm{min})\end{array}$ & $\begin{array}{c}1.3 \pm 0.79 \\
(0.46-3.61)\end{array}$ & 59.85 & $\begin{array}{c}1.5 \pm 1.04 \\
(0.48-4.43)\end{array}$ & 71.06 & 24.11 & 5.65 \\
\hline
\end{tabular}

$\mathrm{N}$ - Number of observations

IIV - Inter-individual variability

IOV - Inter-occasion variability - intra-subject variability was considered as inter-occasion variability

$\dagger \mathrm{N}=24$, since subject no. 13 did not exhibit log-linear elimination in time vs concentration profile

Table 3: Mean obtained pharmacokinetic parameters and inter-occasion, inter-individual and residual variability for Trospium.

Moreover, we did not observe any evidence of enterohepatic circulation or multiple peak in time versus plasma concentration of trospium as verified from profile of individual subject.

According to our data, two way crossover studies following repeated dose administration would be appropriate to define IOV. Results from this article evidencing that when inter-occasion variability not taken into account, may result in the biased parameter estimate. As a concluding remark these problems can be overcome by apparent modeling of IOV.

\section{Conclusion}

Till date for Trospium several population pharmacokinetic models and covariate models have been described. In most cases, first-order absorption and sometimes an absorption lag time was selected to describe the data. In this study non-compartmental model was adopted to evaluate the variability related to Trospium treatment occasion in healthy male subjects. Trospium was well tolerated in the treated subjects and there was no serious adverse event noted in the entire study. Thus, major differences in obtained variability seen in this study are likely to be of only limited clinical significance.

\section{References}

1. Trospium chloride Clinical Pharmacology and Biopharmaceutics Review(s): Application Number 21-595

2. Langguth $P$, Alfons K, Gunter K, Werner L, Merkle, et al. (1997) Intestinal absorption of the quaternary trospium chloride: permeability-lowering factors and bioavailabilities for oral dosage forms. Eur J Pharm Biopharm 43:265-272.

3. Public Assessment Report, Trospasyt 20 mg Filmtablette: Procedure number DE/H/0910/001/DC.

4. Public Assessment Report, DCP, Trospium Chloride 20mg Film-Coated Tablets: UK/H/4220/001/DC.

5. Harnett MD, Shipley J, MacLean L, Schwiderski U, Sandage BW Jr (2013) Study of the Population Pharmacokinetic Characteristics of Once-Daily
Trospium Chloride $60 \mathrm{mg}$ Extended-Release Capsules in Patients with Overactive Bladder and in Healthy Subjects. Clin Drug Investig 33:133-141.

6. SANCTURA ${ }^{\otimes}$ (trospium chloride) $20 \mathrm{mg}$ tablets prescribing information, Allergan, Inc., Revised: 01/2011.

7. Guay DR (2005) Trospium chloride: an update on a quaternary anticholinergic for treatment of urge urinary incontinence. TherClin Risk Manag 1: 157-167.

8. Doroshyenko O, Jetter A, Odenthal KP, Fuhr U (2005) Clinical Pharmacokinetics of Trospium Chloride. ClinPhormocokinet 44:701-720.

9. Nestorov I, Gueorguieva I, Jones HM, Houston B, Rowland M (2002) Incorporating Measures of Variability and Uncertainty into the Prediction of in Vivo Hepatic Clearance from in Vitro Data. Drug Metab Dispos 30: 276-282.

10. Mould DR, Upton RN (2012) Basic Concepts in Population Modeling, Simulation, and Model-Based Drug Development. CPT PharmacometricsSystPharmacol1.

11. Upton RN, Mould DR (2013) Basic Concepts in Population Modeling, Simulation, and Model-Based Drug Development: Part 3-Introduction to Pharmacodynamic Modeling Methods. CPT PharmacometricsSystPharmacol3.

12. Karlsson MO, Sheiner LB (1993) The Importance of Modeling Interoccasion variability in Population Pharmacokinetic Analyses. J Pharmacokinet Biopharm $21: 735-750$

13. Jamei M, Dickinson GL, Rostami-Hodjegan A (2009) A Framework for Assessing inter-individual Variability in Pharmacokinetics Using Virtual Human populations and Integrating General Knowledge of Physical Chemistry, Biology, Anatomy, Physiology and Genetics: A Tale of 'Bottom-Up' vs 'TopDown' Recognition of Covariates. Drug Metab Pharmacokinet 24:53-75.

14. Schröder S, Jetter A, Zaigler M, Weyhenmeyer R, Krumbiegel G, et al. (2004) Absorption pattern of trospium chloride along thehuman gastrointestinal tract assessed using local enteral administration. Int J Clin PharmacolTher 42:543549

15. Albrecht H, Bruhn R, Lorenz D, Lücker PW, Schumacher M (1983) Pupillometry: a noninvasive pharmacokinetic and pharmacodynamic method to study the action of trospium chloride (Spasmolyt $₫$ ) on smooth muscle. Methods Find Exp Clin Pharmacol 5:585-587.

16. Schladitz-Keil G, Spahn H, Mutschler E (1986) Determination of the bioavailability of the quaternary compound trospium chloride in man from urinary excretion data. Arzneimillel Forschung 36: 984-987. 